\title{
Prédiction du comportement dynamique du système de forage grande vitesse vibratoire
}

\author{
Fabien Forestier ${ }^{1, a}$, Vincent Gagnol ${ }^{2}$, Pascal Ray $^{2}$ et Henry Paris ${ }^{3}$ \\ 1 Clermont Université, IUT Montlucon, EA 3867, Laboratoire de Mécanique et Ingénierie, BP 2235, 03101 Montluçon, France \\ 2 Clermont Université, IFMA, EA 3867, Laboratoire de Mécanique et Ingénierie, BP 10448, 63000 Clermont-Ferrand, France \\ ${ }^{3}$ G-SCOP Laboratory, G-SCOP, 46 Av. Félix Viallet, 38031 Grenoble, France
}

Reçu le 25 février 2010, accepté le 6 mai 2010

\begin{abstract}
Résumé - Le Forage Grande Vitesse Vibratoire (FGVV) est un nouveau procédé de perçage qui permet de tripler la productivité des perçages profonds en supprimant les cycles d'évacuation des copeaux ainsi que la lubrification. Pour cela un porte-outil spécifique a été développé, qui entre en vibrations axiales autoentretenues lors de la coupe et permet le fractionnement du copeau, facilitant ainsi son évacuation. Pour anticiper une éventuelle diminution de la durée de vie des roulements à billes de l'électrobroche résultant de ces vibrations, un modèle global du comportement dynamique du système tête vibratoire-électrobroche est présenté. Ce modèle est basé sur la modélisation du comportement des entités structurales par des élémentsfinis de type «poutre-rotor » prenant en compte les effets dynamiques associés aux hautes fréquences de rotations de l'électrobroche, couplé à des modèles d'interfaces identifiés par la méthode de couplage de réceptance. Le modèle est validé à l'aide d'expérimentations qui montrent une bonne correspondance entre les résultats expérimentaux et numériques. Le modèle est ensuite utilisé pour calculer la durée de vie des roulements à billes de l'électrobroche lors de différentes opérations de perçage. L'influence de la fréquence de rotation et de l'avance sur la durée de vie des roulements est étudiée, permettant ainsi de faire ressortir des plages de fonctionnement optimal.
\end{abstract}

Mots clés : Forage grande vitesse vibratoire / comportement dynamique / couplage de réceptance des interfaces / identification

\begin{abstract}
Dynamic behavior model of a high speed vibratory drilling system. High speed vibratory drilling increases the productivity of small diameters deep holes without retreat cycles and lubricant. A specific tool-holder which induces self-sustained vibrations of the mobile part of the vibratory head, breaks up chips and ensures their disposal. To anticipate potential risks associated with these vibrations, a global model of the dynamic behavior of the system (head vibratory-spindle) will be performed. This model is based on the modelisation of the dynamic behavior of the entities throught a rotor-beam element which takes into account the gyroscopic effect and centrifugal forces; associated with interface modelisation identified throught the receptance coupling method. The development and the validation of the dynamic behavior model of the entity are presented. Then, the identification procedure of the interface's dynamic behavior is exposed. Finally, the assembled model is obtained, and used for bearings lifespan prediction during machining. The influence of spindle's speed revolution and machine's feed rate on the rolling bearings lifespan is highlighted. Optimized cutting conditions recommandations are made.
\end{abstract}

Key words: Machine tools dynamics / contact dynamics / parameter identification / receptance coupling / vibratory drilling

\section{Introduction}

Le Forage Grande Vitesse Vibratoire a pour objectif principal d'utiliser des vibrations axiales auto-entretenues

\footnotetext{
a Auteur pour correspondance :

fabien.forestier@moniut.univ-bpclermont.fr
}

pour briser les copeaux. L'instabilité dynamique qui est générée naturellement au cours de la coupe est amplifiée du fait de la faible raideur axiale de la tête. L'amplitude des oscillations du foret est alors supérieure à l'avance par dent ce qui permet la fragmentation des copeaux. Les copeaux étant de petite taille, ils peuvent être évacués sans 
cycle de débourrage ni lubrification. Le forage vibratoire permet ainsi d'augmenter la productivité d'un facteur 3 par rapport aux techniques traditionnelles de perçage. Cependant, l'excitation générée par la coupe peut aussi être une source d'endommagement de l'électrobroche. C'est pourquoi l'industrialisation du procédé de forage grande vitesse vibratoire nécessite de prédire les conséquences des vibrations sur la machine et plus particulièrement sur les électrobroches. Dans ce but, un modèle du comportement dynamique de l'ensemble (tête vibratoire-électrobroche UGV) est élaboré à partir d'un assemblage réaliste des principaux composants de ce système. Cet article présente l'élaboration de ce modèle, en s'intéressant tout particulièrement à l'identification du comportement dynamique des interfaces reliant les principales entités entre elles. Les résultats de simulation du modèle sont ensuite exploités pour prédire la durée de vie des roulements à billes de l'électrobroche en fonction des conditions de coupe.

Une analyse bibliographique a été menée sur la modélisation du comportement dynamique des électrobroches, car elles sont le composant principal du système d'étude (tête vibratoire-électrobroche UGV). Les différents modèles présents dans la littérature peuvent être classés en fonction des paramètres physiques retenus pour étudier le comportement dynamique de l'électrobroche. On trouve ainsi des travaux prenant en compte l'influence de divers paramètres sur le comportement dynamique de l'électrobroche. Parmi ces paramètres, les plus étudiés sont : les effets thermiques [1], la fréquence de rotation de l'électrobroche $[2,3]$, le comportement de la structure de la machine outil [4] et la rigidité des roulements à billes [5]. Ces paramètres sont ceux dont l'influence sur le comportement dynamique de l'électrobroche est la plus importante.

Une attention particulière a été portée sur l'identification du comportement dynamique des interfaces. En effet, le porte-outil spécifique développé pour le FGVV possède une liaison interne dont l'influence est prépondérante sur le comportement dynamique du système global. Les travaux portant sur l'identification du comportement dynamique des interfaces peuvent être classés en trois grandes catégories en fonction de la méthode utilisée.

On trouve ainsi les méthodes dites modales [6,7] qui, à partir de la connaissance des fréquences propres et des déformées modales, permettent de déterminer le comportement des interfaces. Les principaux inconvénients de cette méthode sont d'ordres expérimentaux. En effet, des difficultés apparaissent si les modes propres de la structure sont fortement amortis et/ou couplés. De plus, en fonction des points accessibles sur le système, il peut s'avérer compliqué de récupérer de façon précise les déformées modales.

On trouve aussi les méthodes temporelles [8, 9], qui utilisent les équations du mouvement de la structure pour identifier le comportement de l'interface, et en particulier la méthode de «Force state mapping » $[10,11]$. Les avantages de cette méthode viennent de sa capacité à identifier des comportements fortement non-linéaires. Par contre, la mise en œuvre de la méthode suppose que la masse de la structure soit connue et qu'un nombre important de données expérimentales soit mesuré.

On trouve enfin les méthodes utilisant les Fonctions de Réponse en Fréquence (FRF) [12,13], en particulier la méthode du couplage de réceptance [14-19]. Le principe de l'identification du comportement dynamique des interfaces grâce à cette dernière méthode repose sur le fait que la différence entre le comportement dynamique d'un système assemblé et le comportement dynamique de chacun de ses composants est due à l'interface. Cette méthode a été retenue car elle permet d'identifier des propriétés dynamiques même si les points de l'interface ne sont pas directement accessibles. Parmi les travaux relatifs au couplage de réceptance, on trouve là encore différentes approches. Ren et al. [15] proposent de résoudre un système d'équations issu du couplage de réceptance faisant intervenir un traitement du signal qui minimise l'influence des erreurs de mesure. Schmitz et al. [16] et Movahhedy et al. [17] ramènent l'identification à un problème d'optimisation non-linéaire sous contraintes. Enfin, Erturk et al. [5] et Ozsahin et al. [19] étudient l'influence des rigidités de différentes interfaces dans le but de comprendre le rôle de chacune pour ensuite pouvoir les identifier, obtenant ainsi une nouvelle méthode d'indentification.

Dans l'article proposé, les effets dus à la fréquence de rotation de l'électrobroche sont pris en compte, car le FGVV requiert des vitesses de rotation de l'électrobroche pouvant être supérieures à 10000 tr.min ${ }^{-1}$. La rigidité des roulements à billes est aussi prise en compte car son influence sur le comportement dynamique des broches est importante. Enfin, le comportement dynamique des interfaces est lui aussi intégré dans le modèle car il modifie de façon importante le comportement dynamique $\mathrm{du}$ système global, à basse fréquence. Les effets thermiques sont négligés. Le modèle numérique représente le système à sa température établie d'utilisation. Les dilatations thermiques des pièces, résultant de la mise en service du système sont terminées. Cela se traduit au niveau expérimental par des essais conduits une fois les cycles de préchauffe de la machine effectués. Enfin, le comportement dynamique de la structure de la machine outil n'est pas pris en compte du fait de sa rigidité importante par rapport à celle du système (tête vibratoire-électrobroche UGV).

Après avoir présenté le système d'étude (tête vibratoire-électrobroche UGV), les équations du « couplage de réceptance »sont établies et appliquées. Les modèles sont ensuite validés par comparaison des FRFs numériques et expérimentales. Ensuite, les modèles des entités sont assemblés en intégrant le comportement des interfaces pour obtenir le modèle du comportement dynamique du système global. Après validation du modèle, il est exploité pour déterminer la durée de vie des roulements à billes de l'électrobroche en fonction des conditions de coupe choisies, permettant ainsi de faire ressortir des plages de fonctionnement optimales. 


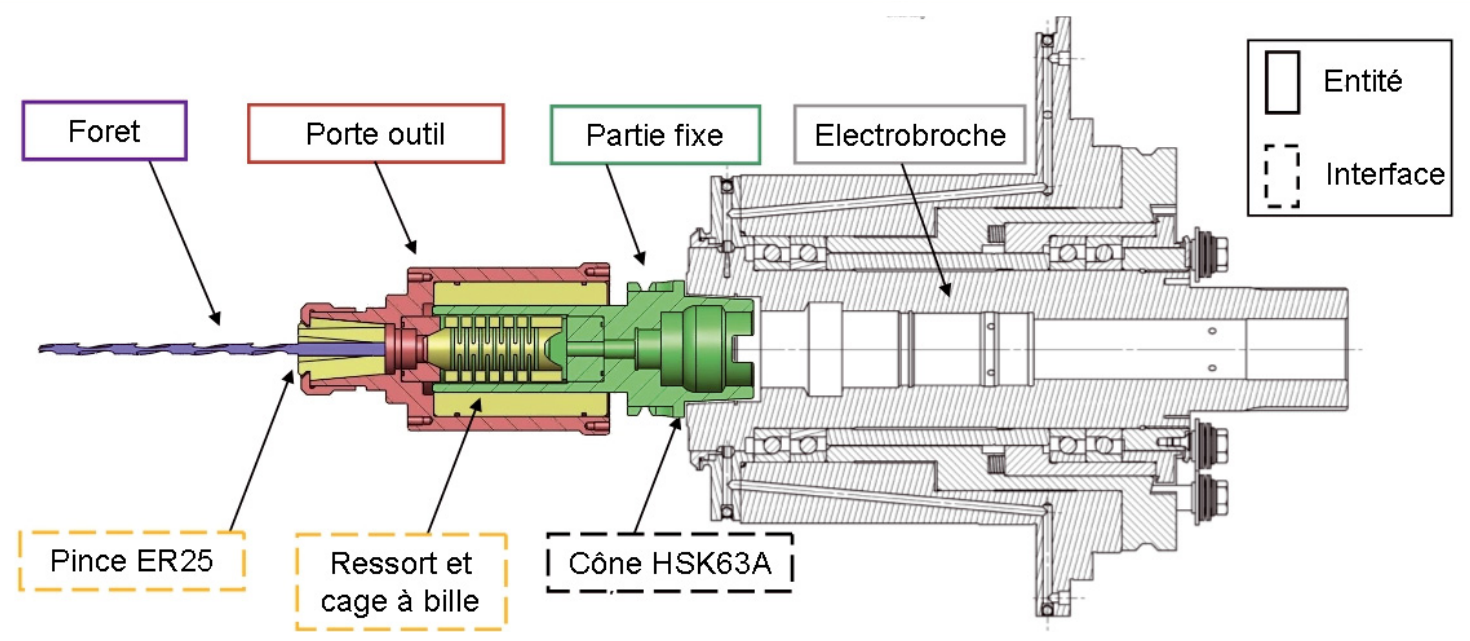

Fig. 1. Décomposition du système (tête vibratoire-électrobroche).

\section{Analyse technologique du système}

Le système (tête vibratoire-électrobroche UGV), représenté sur la figure 1, est constitué des principaux composants suivants :

- Le foret, solidaire de la masse mobile via une pince d'attachement,

- La partie mobile de la tête vibratoire qui est une masse porte-outil animée de vibrations axiales,

- La partie fixe de la tête vibratoire comprenant d'un côté le cône HSK63A et de l'autre le guidage de la liaison glissière avec la partie mobile,

- L'électrobroche.

Ces composants sont reliés entre eux par des interfaces :

- La pince d'attachement ER25 qui permet d'encastrer le foret dans la partie mobile,

- Le ressort et la douille à billes qui assurent la liaison glissière entre la partie mobile et la partie fixe de la tête vibratoire,

- Le cône HSK63A qui rend solidaire la partie fixe de la tête vibratoire avec l'électrobroche.

À partir de cette décomposition du système réel, l'élaboration du modèle numérique est effectuée en trois étapes :

1. L'identification du comportement dynamique des interfaces par la méthode du « couplage de réceptance »,

2. La modélisation du comportement dynamique des entités grâce à un élément-fini « poutre-rotor »,

3. L'assemblage des modèles des entités, en prenant en compte le comportement dynamique des interfaces.

\section{Modélisation et identification du comportement dynamique des interfaces}

\subsection{Présentation de la méthode de couplage de réceptance}

La notation

$$
\underline{\mathbf{A}_{i j}}(\omega)=\left.\frac{\underline{x_{a i}}(\omega)}{\underline{F_{a j, \operatorname{Ext} \rightarrow \mathrm{A}}}(\omega)}\right|_{F_{k, \text { Ext } \rightarrow \mathrm{A}}=0, \forall k \neq j}
$$

désigne la fonction de transfert complexe, appelée réceptance $\mathrm{du}$ système $\mathrm{A}$, dont la sortie est un déplacement en $i$ et l'entrée un effort en $j$. Comme l'ont montré les nombreux travaux utilisant cette méthode, en exprimant le déplacement des extrémités des soussystèmes $\mathrm{A}$ et $\mathrm{B}$, puis du système $\mathrm{AB}$ en fonction des efforts extérieurs et de conditions d'assemblage et de compatibilité, on peut exprimer le comportement dynamique du système assemblé $\mathbf{A B}_{i j}$ en fonction du comportement dynamique de ses composants $\mathbf{A}_{i j}, \mathbf{B}_{i j}$ et de l'interface H (cf. Éqs. (1)-(5)). La figure 2 schématise l'application de la méthode à l'interface reliant la partie mobile et la partie fixe de la tête vibratoire.

$$
\begin{aligned}
\mathbf{A B}_{11} & =\mathbf{A}_{11}-\frac{\mathbf{A}_{12} \mathbf{A}_{21}}{\mathbf{A}_{22}+\mathbf{B}_{11}+\mathbf{H}} \\
\mathbf{A B}_{21} & =\frac{\mathbf{A}_{21} \mathbf{B}_{21}}{\mathbf{A}_{22}+\mathbf{B}_{11}+\mathbf{H}} \\
\mathbf{A B}_{22} & =\mathbf{B}_{22}-\frac{\mathbf{B}_{12} \mathbf{B}_{21}}{\mathbf{A}_{22}+\mathbf{B}_{11}+\mathbf{H}} \\
\mathbf{A B}_{12} & =\frac{\mathbf{A}_{12} \mathbf{B}_{12}}{\mathbf{A}_{22}+\mathbf{B}_{11}+\mathbf{H}}
\end{aligned}
$$

avec $\mathbf{H}$ définie par :

$$
x_{h 1}-x_{h 2}=\mathbf{H} F_{h 2, B \rightarrow H}
$$




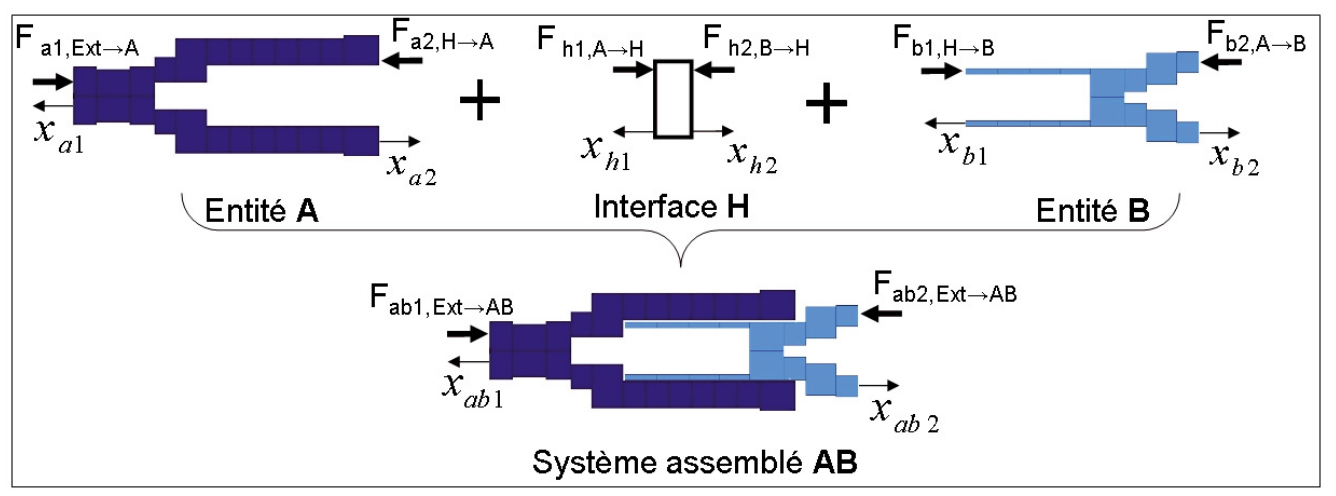

Fig. 2. Méthode du couplage de réceptance appliquée à deux solides reliés par une interface.

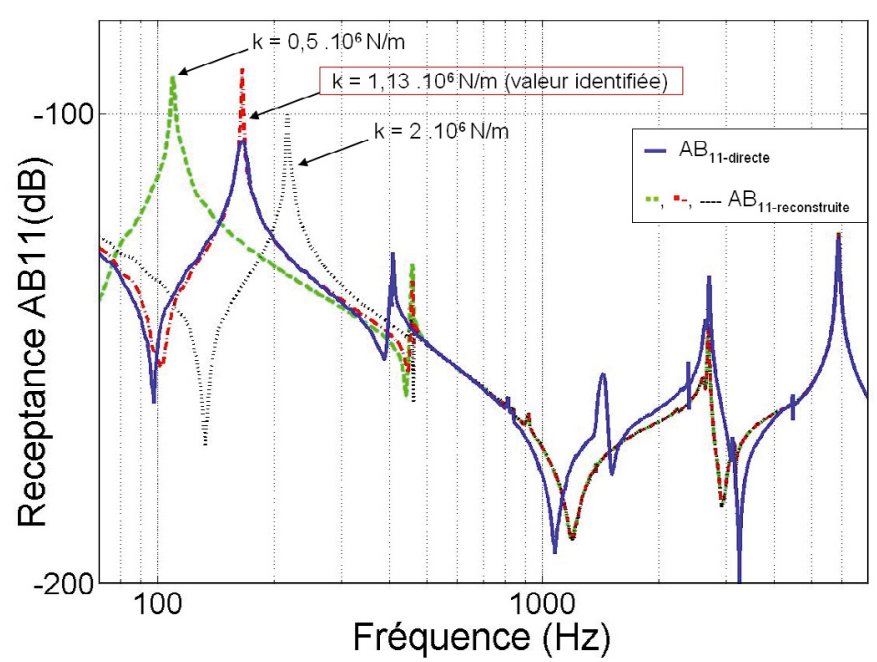

Fig. 3. Identification de $k$ à partir du recalage du pic de résonance de $\mathbf{A} \mathbf{B}_{11-\text { reconstruite à la même fréquence que ce- }}$ lui de $\mathbf{A B}_{11-\text { directe }}$.

\subsection{Application de la méthode de couplage de réceptance à l'identification du comportement dynamique de l'interface reliant la partie mobile et la partie fixe de la tête vibratoire}

Le comportement dynamique axial de l'interface, reliant la partie mobile de la tête vibratoire à la partie fixe, est modélisé par un ressort couplé à un amortisseur en parallèle : $\mathbf{H}_{\text {modele }}=\frac{1}{k+\imath \omega c}$. L'interface est modélisée par un encastrement dans toutes les autres directions. Cette modélisation est justifiée par la réalisation technologique de la liaison qui implique une rigidité axiale beaucoup plus faible que la rigidité obtenue dans les autres directions.

L'équation (1) est utilisée pour identifier le comportement dynamique axial de l'interface : $\mathbf{A B}_{11-\text { reconstruite }}$ désigne la réceptance obtenue grâce à la formule (1) à partir de la réceptance $\mathbf{H}_{\text {modele }}$ et des réceptances mesurées expérimentalement sur les entités A (partie mobile) et B (partie fixe). $\mathbf{A B}_{11-\text { directe }}$ désigne la réceptance mesurée

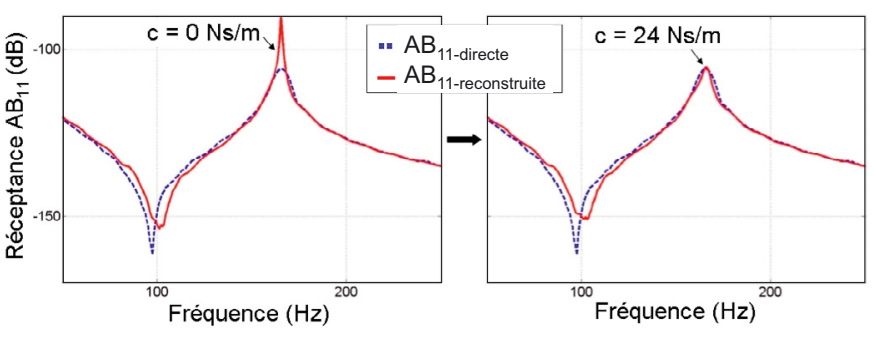

Fig. 4. Identification de $c$ par minimisation de l'écart quadratique entre les courbes $\mathbf{A} \mathbf{B}_{11-\text { reconstruite }}$ et $\mathbf{A B}_{11 \text {-directe. }}$.

directement sur le système assemblé. L'identification des variables $k$ et $c$ est réalisée en deux temps :

- La raideur du ressort $k$ est identifiée en recalant le pic de résonance de la fonction $\mathbf{A B}_{11-\text { reconstruite à la }}$ même fréquence que celui de la fonction $\mathbf{A B}_{11-\text { directe }}$. Les résultats de cette identification sont présentés sur la figure 3 .

- Le facteur d'amortissement $c$ est ensuite identifié dans le but de minimiser l'écart en amplitude entre la courbe $\mathbf{A} \mathbf{B}_{11-\text { reconstruite }}$ et la courbe $\mathbf{A B}_{11-\text { directe, }}$ comme le montre la figure 4 .

Les valeurs obtenues pour la rigidité et le facteur d'amortissement de la liaison reliant la partie mobile à la partie fixe de la tête vibratoire sont respectivement : $437 \times$ $10^{3}$ N.m ${ }^{-1}$ et 24 N.s.m ${ }^{-1}$.

\subsection{Application de la méthode de couplage de réceptance à l'identification du comportement dynamique de l'interface : pince ER25}

La liaison entre le foret et la partie mobile de la tête vibratoire est un encastrement réalisé par une pince expansible. La rigidité axiale est supposée beaucoup plus faible que la rigidité obtenue dans les autres directions. Le comportement dynamique de cette interface est donc modélisé axialement par un système ressort-amortisseur et dans toutes les autres directions par des encastrements. 


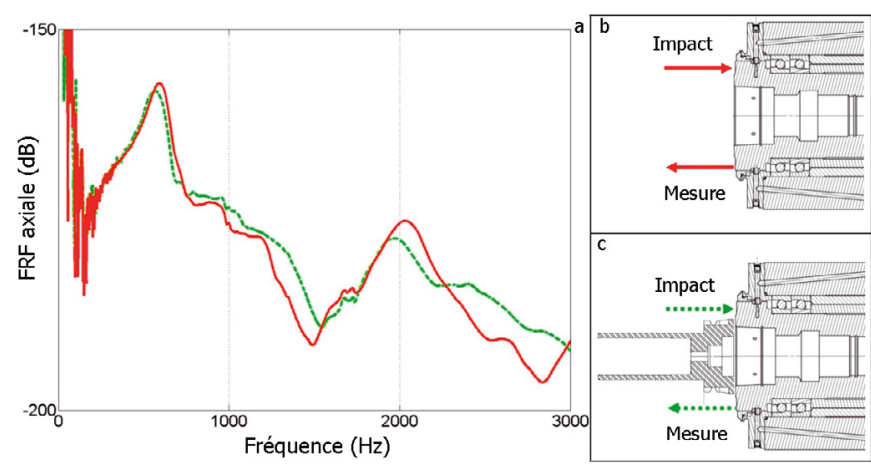

Fig. 5. (a) FRF, dans la direction axiale, en nez de broche avec et sans la partie fixe de la tête vibratoire. (b) Configuration de l'essai de sonnage de l'électrobroche. (c) Configuration de l'essai de sonnage de l'électrobroche avec la partie fixe de la tête vibratoire.

La méthode de couplage de réceptance est à nouveau utilisée pour identifier la rigidité et l'amortissement du modèle ressort-amortisseur. Cependant la

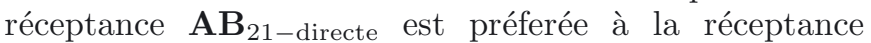
$\mathbf{A B}_{11-\text { directe, }}$ car il est difficile de fixer correctement un accéléromètre à l'extrémité du foret. De plus, la réceptance $\mathbf{A B}_{21-\text { reconstruite est maintenant obtenue }}$ grâce à la formule (2) à partir de la réceptance $\mathbf{H}_{\text {modele }}$ et des réceptances numériques des modèles des entités $\mathrm{A}$ (foret) et B (partie mobile). En effet, la masse de l'accéléromètre n'étant pas négligeable par rapport à la masse du foret, les analyses modales expérimentales du foret sont peu précises [20]. De même, les sonnages au marteau de la partie mobile ne donnent pas de résultats satisfaisants lorsque la pince d'attachement de cette dernière ne sert pas d'outil. C'est pourquoi, pour ces deux entités, des réceptances numériques ont été préférées aux réceptances expérimentales.

La procédure d'identification de $k$ et $c$ reste identique à celle présentée dans la section 3.2. Les valeurs obtenues pour la rigidité et le facteur d'amortissement de l'interface reliant le foret à la partie mobile de la tête vibratoire sont respectivement : $14,8 \times 10^{6} \mathrm{~N} . \mathrm{m}^{-1}$ et 4 N.s.m ${ }^{-1}$.

\subsection{Identification du comportement dynamique de l'interface réalisée par le cône HSK63A}

L'identification du comportement dynamique de l'interface réalisée par le cône HSK63A est traitée différemment. Des essais menés à la fois sur le système (partie fixe de la tête vibratoire-électrobroche) et sur le système (électrobroche seule) montrent que le comportement dynamique axial de ces deux systèmes est identique (cf. Fig. 5). À partir de ces observations, l'interface réalisée par le cône HSK63A est modélisée par un encastrement. Dans la suite de l'article, la partie fixe de la tête vibratoire et l'électrobroche seront considérées comme une seule et même entité.

\section{Modélisation et validation du comportement dynamique des entités}

\subsection{Modèle d'entités}

La figure 6 représente le modèle numérique du système complet. Le comportement dynamique des entités est modélisé par la méthode des éléments-finis en utilisant un élément «poutre-rotor » spécifique [2]. Cet élément possède deux nœuds, reliés par des fonctions de forme cubique, et six degrés de liberté par noeud. Cet élément prend en compte les forces centrifuges et les effets gyroscopiques apparaissant dans les entités en rotation. Pour l'électrobroche, un modèle de rigidité non-linéaire des roulements à billes, basé sur les travaux de Lim et Singh [21] est introduit.

Le modèle numérique repose sur les équations différentielles de la dynamique des rotors (cf. Éq. (6)) :

$$
\mathbf{M}\left(\mathbf{q}_{N}\right) \ddot{\mathbf{q}}_{N}+\left(\mathbf{C}\left(\mathbf{q}_{N}, \dot{\mathbf{q}}_{N}\right)+\mathbf{D}\right) \dot{\mathbf{q}}_{N}+\mathbf{K} \mathbf{q}_{N}=\mathbf{F}(t)
$$

avec $\mathbf{M}\left(\mathbf{q}_{N}\right)$ et $\mathbf{K}$ les matrices de masse et de rigidité. $\mathbf{C}\left(\mathbf{q}_{N}, \dot{\mathbf{q}}_{N}\right)$ est une matrice contenant les effets dynamiques dus à la rotation du système. $\mathbf{D}$ est la matrice d'amortissement qui est une combinaison linéaire des matrices de masse et de rigidité : $\mathbf{D}=\alpha \mathbf{K}_{\text {broche }}+\beta \mathbf{M}+$ $\gamma \mathbf{K}_{\text {roulements }}$ (amortissement de Rayleigh). $\mathbf{q}_{N}$ et $\mathbf{F}(t)$ sont les déplacements nodaux et le vecteur des efforts extérieurs.

\subsection{Validation}

La partie fixe de la tête vibratoire et l'électrobroche sont considérées comme une unique entité. Les modèles du foret et de la partie mobile sont validés une fois assemblés. En effet, l'analyse modale du foret seul n'est pas précise car elle est faussée par la masse des accéléromètres [20] ; d'autre part les sonnages au marteau de la partie mobile ne donnent pas de résultats satisfaisants lorsque la pince d'attachement de cette dernière ne sert pas d'outil. Les entités dont le comportement doit être validé sont donc la partie fixe de la tête vibratoire et l'électrobroche; le foret et la partie mobile de la tête vibratoire.

La comparaison des FRFs numériques et des FRFs expérimentales est présentée sur les figures 7 et 8 . Sur ces figures, il apparaît la représentation géométrique des modèles des entités ainsi que les points d'impact du marteau de sonnage et la localisation des accéléromètres.

Les propriétés mécaniques des matériaux (module de Young), la pré-charge des roulements à billes de l'électrobroche et/ou la géométrie (diamètre du foret) servent de variable de recalage des modèles numériques pour minimiser l'écart en fréquence au niveau des pics de résonance entre les FRFs numériques et expérimentales. Les paramètres d'amortissements structuraux des entités permettent de minimiser l'écart en amplitude entre les courbes numériques et expérimentales. La connaissance détaillée d'une broche n'est généralement pas possible en 


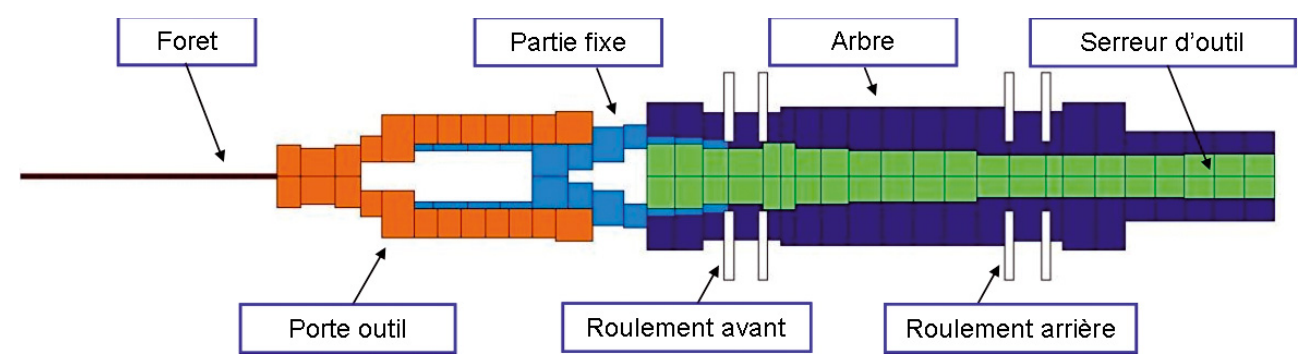

Fig. 6. Modélisation du système complet.

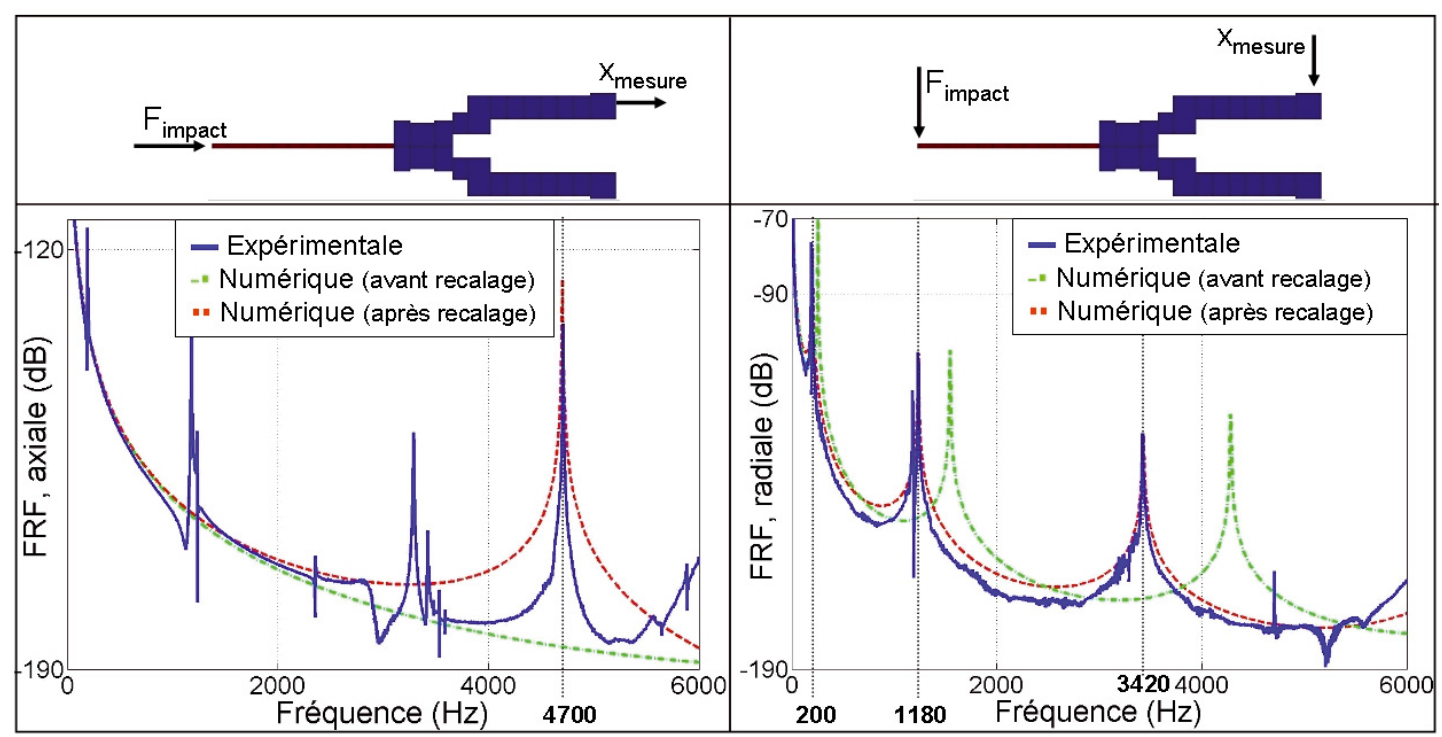

Fig. 7. Schématisation du modèle numérique du foret assemblé à la partie mobile de la tête vibratoire et FRFs numériques et expérimentales de ce système.

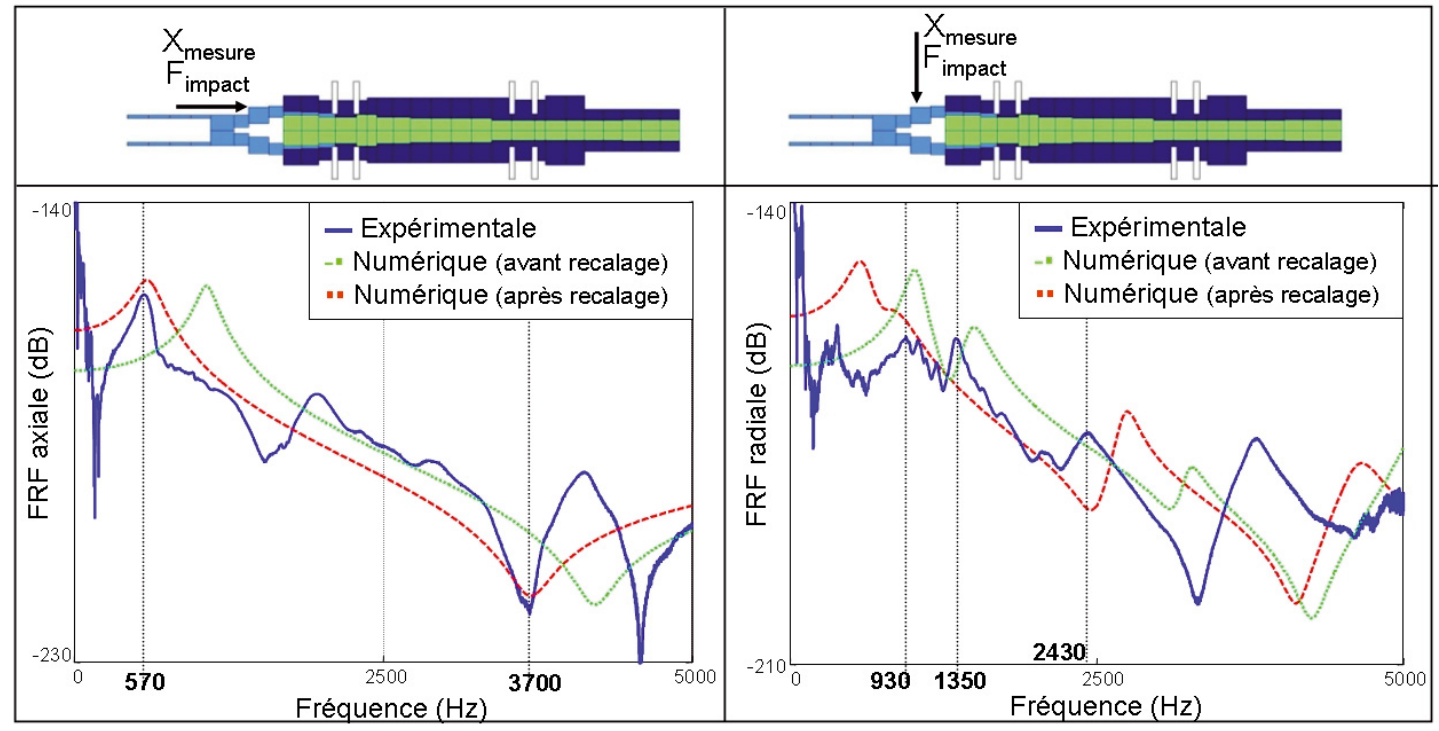

Fig. 8. Schématisation du modèle numérique de la partie fixe de la tête vibratoire encastrée à l'électrobroche et FRFs numériques et expérimentales de ce système. 


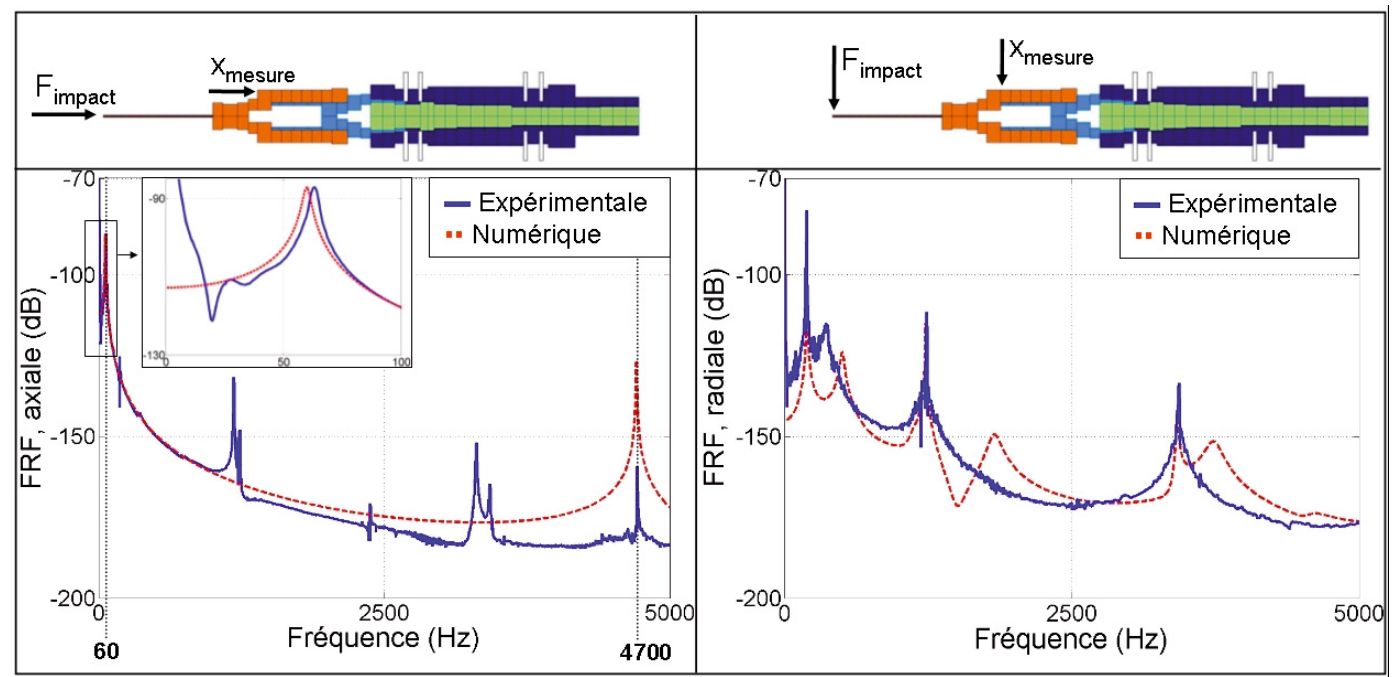

Fig. 9. Schématisation du modèle numérique assemblé et FRFs numériques et expérimentales de ce système.

Tableau 1. Données matérielles du foret et de la partie mobile de la tête vibratoire avant et après recalage.

\begin{tabular}{llcc}
\hline & Entité & $\begin{array}{c}\text { Module } \\
\text { de Young }(\mathrm{Pa})\end{array}$ & $\begin{array}{c}\text { Diamètre du } \\
\text { foret }(\mathrm{mm})\end{array}$ \\
\hline Valeurs avant & Partie mobile & $2 \times 10^{11}$ & \\
recalage & Foret & $6 \times 10^{11}$ & 4 \\
Valeurs après & Partie mobile & $2 \times 10^{11}$ & \\
recalage & Foret & $6 \times 10^{11}$ & 3,29 \\
\hline
\end{tabular}

configuration industrielle. Le modèle doit donc être recalé pour correspondre aux résultats expérimentaux. Les propriétés matériaux du rotor et du serreur d'outil telles que le module de Young ne sont pas connues précisément en raison de la simplification géométrique du modèle et de la complexité géométrique et physique de ces entités. Les tableaux 1 et 2 montrent les valeurs des variables de recalage avant et après modification.

Pour le modèle du foret assemblé à la partie mobile de la tête vibratoire, seul le diamètre du foret a été modifié lors du recalage fréquentiel (cf. Fig. 7). Le foret étant modélisé par un cylindre plein, le diamètre du foret a été retenu comme variable de recalage car c'est le paramètre ayant la plus grande incertitude. Les pics à 200, 1180 et $3420 \mathrm{~Hz}$ n'apparaissent pas sur les FRFs axiales numériques, car ils sont dus à des modes de flexion. Les différences entre les courbes avant et après recalage mettent en évidence l'influence du diamètre du foret sur les FRFs radiales et l'influence de la rigidité de la liaison entre le foret et la partie mobile de la tête vibratoire sur les FRFs axiales. En effet sur les courbes avant réajustement, un foret de $4 \mathrm{~mm}$ de diamètre est encastré à la partie mobile, alors que sur les courbes après réajustement, un foret de diamètre $3,29 \mathrm{~mm}$ est relié à la partie mobile via la rigidité identifiée de l'interface.

Pour le modèle de l'électrobroche assemblée à la partie fixe de la tête vibratoire, le premier pic de résonance, en traction-compression, à $570 \mathrm{~Hz}$ est principalement contrôlé par la valeur de la pré-charge des roulements à billes de l'électrobroche. La fréquence du premier pic d'anti-résonance, à $3700 \mathrm{~Hz}$, a été recalée grâce au module de Young du rotor de l'électrobroche (cf. Fig. 8). Le recalage du modèle est principalement basé sur la FRF axiale. Une perte de précision s'observe, sur la FRF radiale numérique après recalage par rapport à la FRF avant recalage, au niveau des deux premiers pics expérimentaux à $930 \mathrm{~Hz}$ et $1350 \mathrm{~Hz}$. Par contre le pic expérimental à $2430 \mathrm{~Hz}$ est obtenu de façon plus précise sur la courbe après recalage.

\section{Assemblage du modèle et validation}

Les paramètres dynamiques identifiés, dans la direction axiale, grâce à la méthode de couplage de réceptance, pour la liaison entre le foret et la partie mobile de la tête vibratoire et pour la liaison entre la partie mobile et la partie fixe de la tête vibratoire, permettent de définir les rigidités et les coefficients d'amortissement des éléments ressort-amortisseur. Les modèles éléments finis « poutre-rotor» des composants sont assemblés grâce à ces éléments ressort-amortisseur.

\subsection{Validation du comportement dynamique du système assemblé}

Dans cette partie la validation du modèle assemblé (tête vibratoire-électrobroche) est présentée. Sur la figure 9, les FRFs issues du modèle numérique assemblé sont comparées avec les FRFs expérimentales. À ce stade, aucun recalage n'est effectué sur le modèle numérique.

Sur les FRFs axiales de la figure 9 , à $60 \mathrm{~Hz}$, on peut observer le mode de résonance dû à l'interface, reliant la partie mobile à la partie fixe de la tête vibratoire, pour lequel les courbes expérimentales et numériques coïncident en fréquence et en amplitude. À $4700 \mathrm{~Hz}$, dans la direction axiale, il apparaît le mode dû à l'interface reliant le 
Tableau 2. Données matérielles de la broche et de la partie fixe de la tête vibratoire et précharge des roulements avant et après recalage.

\begin{tabular}{llcc}
\hline & Entité & $\begin{array}{c}\text { Module de Young } \\
(\mathrm{Pa})\end{array}$ & $\begin{array}{c}\text { Précharge des } \\
\text { roulements }(\mathrm{N})\end{array}$ \\
\hline Valeurs avant & (Arbre + Serreur d'outil) & $2 \times 10^{11}$ & 70 \\
recalage & Partie Fixe & $2 \times 10^{11}$ & \\
Valeurs après & (Arbre + Serreur d'outil) & $1,5 \times 10^{11}$ & 8 \\
recalage & Partie Fixe & $2 \times 10^{11}$ & \\
\hline
\end{tabular}

Tableau 3. Fréquences et amplitudes des pics de résonance des FRFs axiales expérimentales et numériques.

\begin{tabular}{ccccc}
\hline & & FRF & FRF & erreur \\
& & numérique & expérimentale & relative (\%) \\
\hline Fréquence $(\mathrm{Hz})$ & mode 1 & 61 & 63 & 3 \\
& mode 2 & 4708 & 4713 & 0,1 \\
Amplitude $(\mathrm{dB})$ & mode 1 & -87 & -87 & 0 \\
& mode 2 & $-126,5$ & -159 & 20 \\
\hline
\end{tabular}

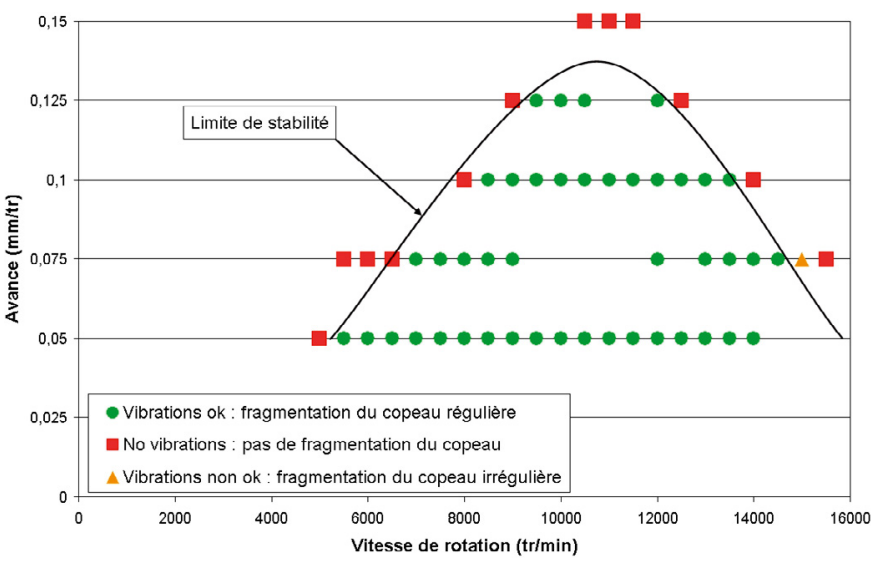

Fig. 10. Essais réalisés présentant le domaine d'instabilité de la coupe en fonction de la fréquence de rotation de l'électrobroche et de la vitesse d'avance.

foret à la partie mobile de la tête vibratoire. Les pics à 200, 1180 et $3420 \mathrm{~Hz}$, sur la FRF axiale expérimentale, sont dûs à des modes de flexion et n'apparaissent donc pas sur la FRF numérique axiale.

Globalement, pour les FRFs axiales et radiales, on observe une bonne correspondance entre les courbes expérimentales et numériques (cf. Tabs. 3 et 4), ce qui permet de valider le modèle numérique assemblé du système (tête vibratoire-électrobroche).

\section{Exploitation du modèle}

Dans cette partie, le modèle numérique est utilisé pour prédire la durée de vie des roulements à billes de l'électrobroche lors d'usinages réalisés avec des conditions de coupe différentes.

Des essais de forage grande vitesse vibratoire ont été effectués pour mesurer les efforts de coupe, via une table de mesures d'efforts (Dynamomètre Kistler Type 9257B), dans différentes conditions de coupes (cf.

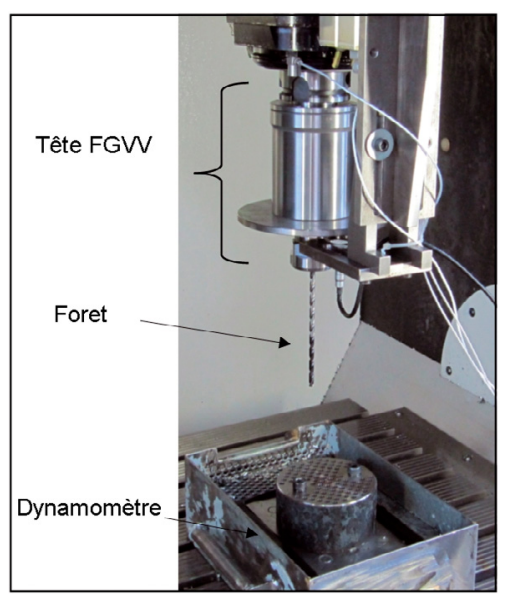

Fig. 11. Dispositif expérimental.

Fig. 10). Ces essais ont été menés sur un centre d'usinage (Centre d'usinage Hermle 3 axes C800U équipé d'une broche Weiss 18000 tr. $\min ^{-1}$ ), dans un acier de nuance $35 \mathrm{MnV} 7$, avec un foret de $116 \mathrm{~mm}$ de longueur utile et de $5 \mathrm{~mm}$ de diamètre. La figure 11 représente le dispositif expérimental.

Le perçage vibratoire nécessite de définir les conditions de coupe dans le domaine instable des lobes de stabilité pour obtenir des vibrations permettant une fragmentation régulière du copeau. Différentes vitesses de rotation de l'électrobroche et vitesses d'avance ont été testées, respectivement entre 5000 et 15500 tr.min $^{-1}$ et entre 0,05 et $0,015 \mathrm{~mm} \cdot \mathrm{tr}^{-1}$. Seuls les usinages ayant conduit à une bonne fragmentation du copeau ont été retenus pour le calcul de la durée de vie des roulements à billes de l'électrobroche (Fig. 10). En particulier, le point correspondant à une fréquence de rotation de l'électrobroche de $15000 \mathrm{tr}^{\mathrm{min}}{ }^{-1}$ et une avance de $0,075 \mathrm{~mm} \cdot \mathrm{tr}^{-1}$ n'est pas retenu car la fragmentation du copeau n'a pas été régulière. 
Tableau 4. Fréquences et amplitudes des pics de résonance des FRFs radiales expérimentales et numériques.

\begin{tabular}{|c|c|c|c|c|}
\hline & & $\begin{array}{c}\text { FRF } \\
\text { numérique }\end{array}$ & $\begin{array}{c}\text { FRF } \\
\text { expérimentale }\end{array}$ & $\begin{array}{c}\text { erreur } \\
\text { relative }(\%)\end{array}$ \\
\hline \multirow[t]{4}{*}{ Fréquence $(\mathrm{Hz})$} & mode 1 & 196 & 193 & 1,5 \\
\hline & mode 2 & 503 & 376 & 25 \\
\hline & mode 3 & 1229 & 1237 & 0,6 \\
\hline & mode 4 & 1830 & & \\
\hline \multirow[t]{5}{*}{ Amplitude (dB) } & mode 1 & -103 & -80 & 22 \\
\hline & mode 2 & -124 & -115 & 7 \\
\hline & mode 3 & -115 & $-111,5$ & 3 \\
\hline & mode 4 & -140 & & \\
\hline & mode 5 & -142 & $-133,5$ & 6 \\
\hline
\end{tabular}

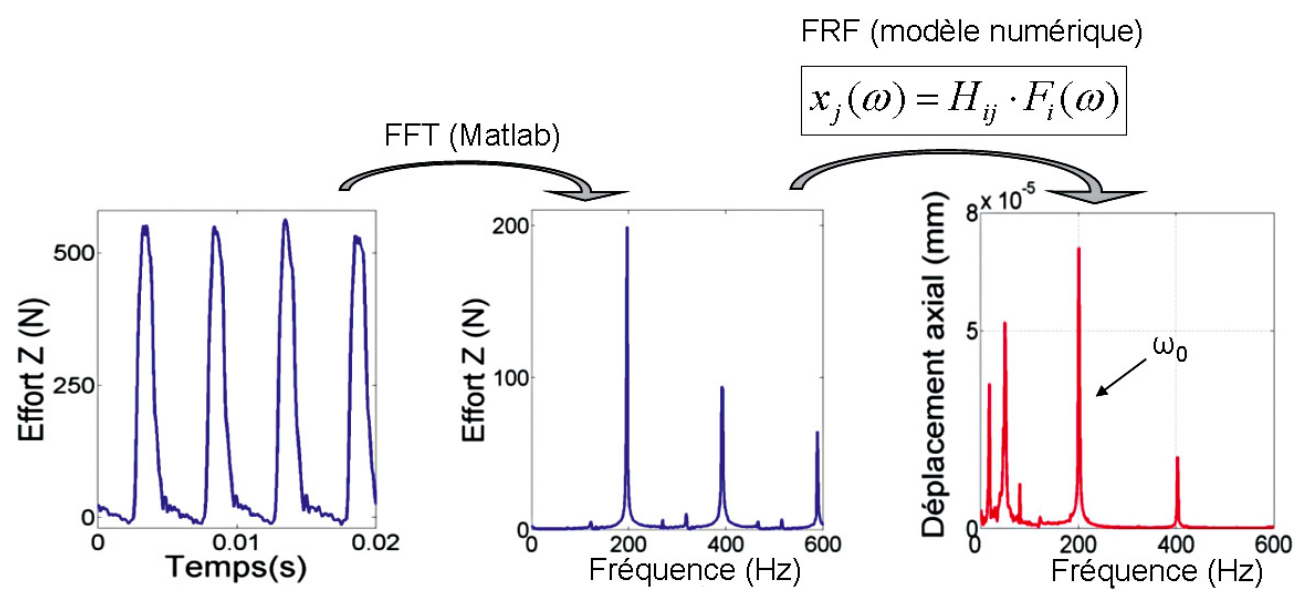

Fig. 12. Méthode de calcul du déplacement du noeud de chaque roulement, dans les trois directions, à partir des efforts de coupe mesurés.

Le modèle numérique du système assemblé est ensuite utilisé pour obtenir les FRFs numériques, à la fréquence de rotation de l'électrobroche, dans les trois directions, entre le bout d'outil et les roulements à billes de l'électrobroche. Il est intéressant de remarquer sur la figure 15 , l'influence de la fréquence de rotation sur le comportement dynamique du système. On peut observer une modification de la fréquence et de l'amplitude des modes de résonance qui s'explique par un découplage gyroscopique qui est pris en compte par l'élément « poutrerotor ». Ce phénomène explique en partie la différence de durée de vie des roulements en fonction des conditions de coupe.

Les efforts de coupe mesurés au niveau de l'outil, dans le domaine fréquentiel, sont multipliés aux FRFs précédentes dans le but d'obtenir le déplacement de chaque roulement, dans le domaine fréquentiel (cf. Fig. 12) :

$\underline{X}(\omega)_{\text {roulement }}=\underline{F R F}\left(\omega, \Omega_{0}\right)_{\text {numerique }} \times \underline{F}(\omega)_{\text {experimentale }}$

L'amplitude de ces déplacements est ensuite calculée à la pulsation de vibration de la tête vibratoire $\omega_{0}$, puis multipliée par la matrice de rigidité du roulement, dans la direction concernée, pour obtenir les efforts axiaux et radiaux appliqués au roulement :

$$
\begin{aligned}
X\left(\omega_{0}\right)_{\text {roulement }}=\mid & \underline{X}(\omega)_{\text {roulement }} \mid \\
& \text { à la pulsation de vibration } \omega_{0} \\
F\left(\omega_{0}\right)_{\text {roulement }}= & \mathbf{K}_{\text {roulement }} \times X\left(\omega_{0}\right)_{\text {roulement }}
\end{aligned}
$$

À partir des efforts axiaux et radiaux, la charge équivalente dynamique, $P$ est calculée. La durée de vie du roulement est calculée à partir de la formule suivante :

$$
L_{h}=\frac{10^{6}}{60 n}\left(\frac{C}{P}\right)^{3}
$$

avec $L_{h}$ la durée de vie du roulement en heures, $C$ la charge dynamique de base du roulement en Newton, $P$ la charge dynamique équivalente du roulement en Newton et $n$ la vitesse de rotation de la broche en tr. $\mathrm{min}^{-1}$.

L'étude de la durée de vie des différents roulements montre que celui qui possède la plus faible durée de vie est toujours le roulement avant. La figure 13 illustre la durée de vie de chaque roulement, calculée pour la condition de coupe la plus sévère obtenue lors des essais. Ce graphique montre que, même dans ce cas, la durée de vie de 


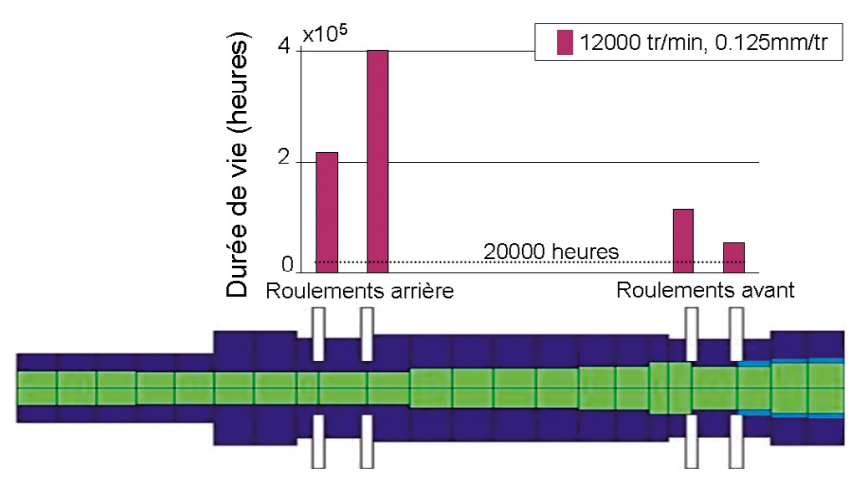

Fig. 13. Durée de vie de chaque roulement calculée dans le cas le plus défavorable.

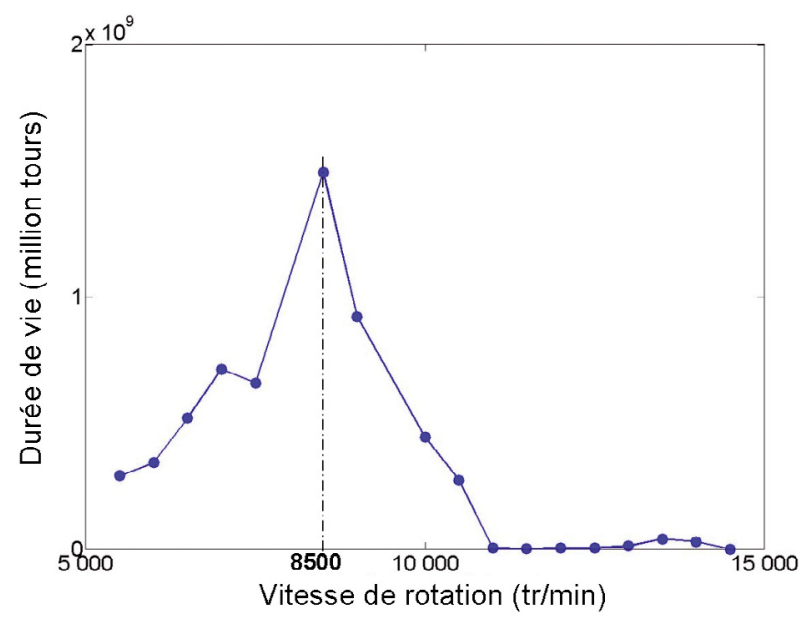

Fig. 14. Durée de vie du roulement avant en fonction de la fréquence de rotation de l'électrobroche pour une avance de $0,05 \mathrm{~mm} \cdot \mathrm{tr}^{-1}$.

tous les roulements est supérieure aux préconisations des constructeurs de broche qui dimensionnent leurs roulements pour une durée de vie de 20000 h. Pour un diamètre de $5 \mathrm{~mm}$, une opération de forage grande vitesse vibratoire n'a donc aucun impact néfaste sur la durée de vie des roulements. Il est à noter que les efforts de coupe peuvent être plus importants pour des diamètres supérieurs de foret.

La figure 14 représente l'évolution de la durée de vie, du roulement avant, en fonction de la fréquence de rotation de l'électrobroche, pour une avance de $0,05 \mathrm{~mm} \cdot \mathrm{tr}^{-1}$. Cette courbe montre qu'il existe une fréquence de rotation optimale, aux alentours de 8500 tr. min $^{-1}$, qui permet de maximiser la durée de vie des roulements. La forme de cette courbe s'explique grâce à la superposition de deux effets. Auparavant, il faut noter que l'amplitude des efforts de coupe est sensiblement la même quelle que soit la vitesse de rotation de la broche, pour une avance fixée.

D'une part, on remarque que les FRFs sont modifiées par la variation de la fréquence de rotation de l'électrobroche liée au découplage gyroscopique pris en compte par l'élément «poutre-rotor » (Fig. 15b). D'autre part, la fréquence de vibration de la tête vibratoire est elle

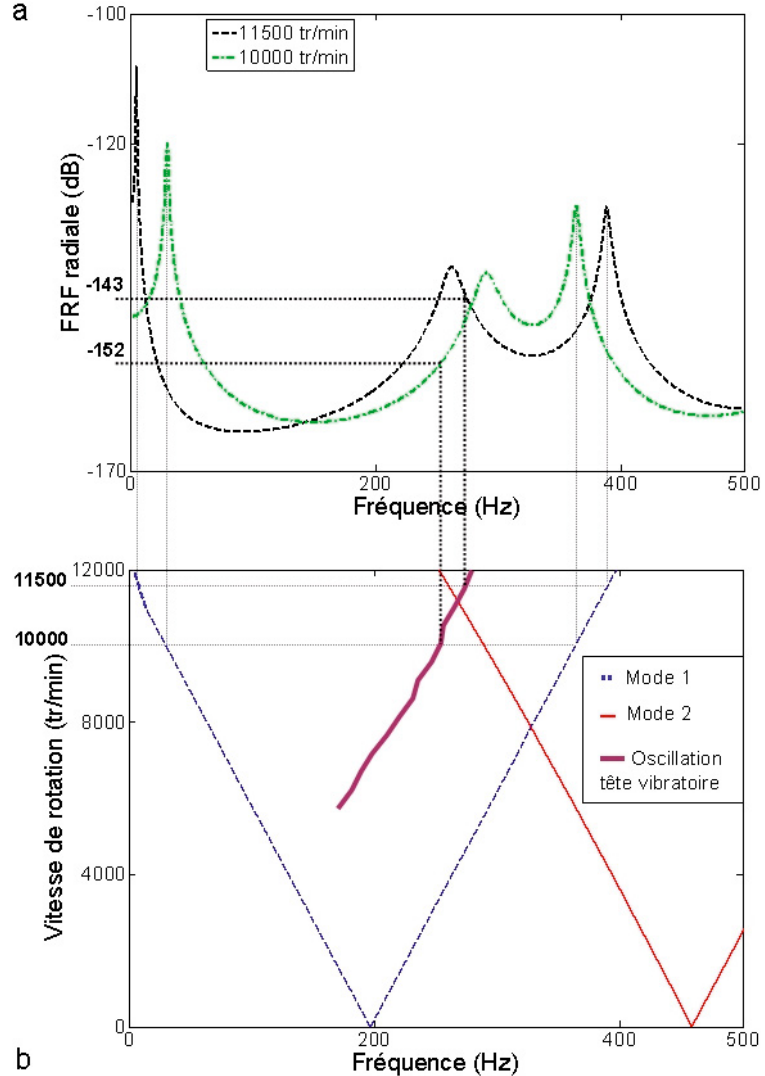

Fig. 15. (a) Amplitude des FRFs à la fréquence d'oscillation de la tête vibratoire. (b) Évolution de la fréquence d'oscillation de la tête vibratoire et des modes propres du système en fonction de la vitesse de rotation de l'électrobroche.

aussi modifiée par la variation des conditions de coupe (Fig. 15b). Or d'après l'équation (8), seule la pulsation d'oscillation de la tête vibratoire intervient dans le calcul de la durée de vie des roulements. La figure 15a représente l'amplitude des FRFs, à la pulsation de vibration de la tête vibratoire, pour deux vitesses de rotation différentes de la broche. Cette figure cumule les deux effets précédents et montre que pour une vitesse de rotation de 10000 tr.min ${ }^{-1}$ les déplacements du roulement avant seront plus faibles que pour une vitesse de rotation de 11500 tr. $\mathrm{min}^{-1}$. Ceci explique que la durée de vie du roulement avant pour une fréquence de rotation de 10000 tr. $\mathrm{min}^{-1}$ est plus élevée que celle observée pour une fréquence de rotation de $11500 \mathrm{tr} \cdot \mathrm{min}^{-1}$.

La figure 16 représente l'évolution de la durée de vie, $\mathrm{du}$ roulement avant, en fonction de l'avance, pour une fréquence de rotation de 12000 tr. $\mathrm{min}^{-1}$. Cette courbe montre que l'avance idéale est de $0,05 \mathrm{~mm} \cdot \mathrm{tr}^{-1}$ et que les avances aux alentours de $0,1 \mathrm{~mm} \cdot \mathrm{tr}^{-1}$ sont à éviter.

\section{Conclusions}

L'étude proposée s'appuie sur la modélisation d'un nouveau procédé de fabrication : le FGVV. Une démarche d'élaboration originale d'un modèle de comportement 


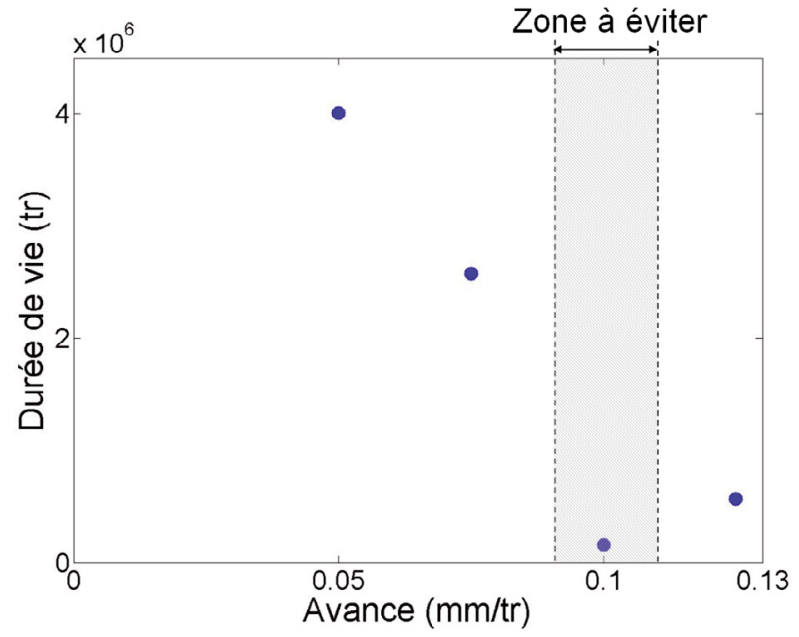

Fig. 16. Durée de vie du roulement avant en fonction de l'avance pour une fréquence de rotation de l'électrobroche de 12000 tr. $\mathrm{min}^{-1}$.

dynamique du système (tête vibratoire-électrobroche) est proposée. Ce modèle repose sur une modélisation «poutre-rotor », pour les entités structurales, couplé à des éléments ressort-amortisseur qui prennent en compte le comportement des interfaces. Chaque étape d'élaboration du modèle a été validée grâce à une comparaison entre des FRFs issues du modèle numérique et des FRFs mesurées expérimentalement. Le comportement dynamique des interfaces a été identifié à partir de données expérimentales grâce à l'utilisation de la méthode de couplage de réceptance. Le modèle obtenu à l'issue de l'assemblage permet de simuler avec une bonne précision le comportement dynamique réel du système. Il a ensuite été utilisé pour prédire la durée de vie des roulements à billes de l'électrobroche, pendant une opération de FGVV. L'exploitation du modèle permet de déterminer les conditions de coupe optimales, qui permettent d'optimiser la tenue en service du système. Il permet aussi de conclure que le FGVV induit une faible sollicitation sur la durée de vie des roulements à billes de l'électrobroche.

\section{Références}

[1] J. Jedrzejewski, Z. Kowal, W. Kwasny, W. Modrzycki, Hybrid Model of High Speed Machining Centre Headstock, Annals of the CIRP - Manufacturing Technology 53 (2004) 285-288

[2] V. Gagnol, C.B. Bouzgarrou, P. Ray, C. Barra, Modelbased chatter stability prediction for high-speed spindles, Int. J. Mach. Tool. Manu. 47 (2007) 1176-1186

[3] Y. Cao, A general method for the modeling of spindlebearing systems, J. Mech. Des. 126 (2004) 1089-1104

[4] Y. Cao, Y. Altintas, Modeling of spindle-bearing and machine tool system for virtual simulations of milling operations, Int. J. Mach. Tool. Manu. 47 (2007) 1342-1350

[5] A. Ertürk, H.N. Özgüven, E. Budak, Effect analysis of bearing and interface dynamics on tool point FRF for chatter stability in machine tools by using a new analytical model for spindle tool assemblies, Int. J. Mach. Tool. Manu. 47 (2007) 23-32

[6] M.R. Good, D.J. Marioce, Using experimental modal analysis to characterize automobile body joints and improve finite element analysis, Proceedings of Seventh International Modal Analysis Conference, Las Vegas, 1989, pp. 106-110

[7] A.S. Nobari, D.A. Robb, D.J. Ewins, A new modalbased method for structural dynamic model updating and joint identification, Proceedings of the 10th International Modal Analysis Conference 1, San Diego, 1992, pp. 741-750

[8] S.F. Masri, T.K. Caughey, A non parametric identification technique for non linear dynamic problems, J. Appl. Mech. 46 (1979) 433-447

[9] S.F. Masri, T.K. Caughey, Non parametric identification of nearly arbitrary non linear systems, J. Appl. Mech. 49 (1982) 619-628

[10] E.F. Crawley, A.C. Aubert, Identification of nonlinear structural elements by force-state mapping, Am. Inst. Aeronautics Astronautics J. 24 (1986) 155-162

[11] E.F. Crawley, K.J. O'Donnell, Force-state mapping identification of nonlinear joints, Am. Inst. Aeronautics Astronautics J. 25 (1987) 1003-1010

[12] J.E. Mottershead, R. Stanway, Identification of structural vibration parameters by using a frequency domain filter, J. Sound Vib. 109 (1986) 495-506

[13] J.S. Tsai, Y.F. Chou, The identification of dynamic characteristics of a single bolt joint, J. Sound Vib. 125 (1988) $487-502$

[14] R.E.D. Bishop, D.C. Johnson, The Mechanics of Vibration, Cambridge University Press, 1960

[15] Y. Ren, C.F. Beards, Identification of joint properties of structure using FRF data, J. Sound Vib. 186 (1995) 567587

[16] T.L. Schmitz, R. Donaldson, Predicting high-speed machining dynamics by a substructure analysis, Annals of CIRP 49 (2000) 303-308

[17] M. Movahhedy, J. Gerami, Prediction of spindle dynamics in milling by sub-structure coupling, Int. J. Mach. Tool. Manu. 46 (2006) 243-251

[18] A. Ertürk, H.N. Özgüven, E. Budak, Analytical modelling of spindle tool dynamics on machine tools using Timoshenko beam model and receptance coupling for the prediction of tool point FRF, Int. J. Mach. Tool. Manu. 46 (2006) 1901-1912

[19] O. Özsahin, A. Ertürk, H.N. Özgüven, E. Budak, A Closed-form approach for identification of dynamical contact parameters in spindle-holder-tool assemblies, Int. J. Mach. Tool. Manu. 49 (2009) 25-35

[20] O. Özsahin, H.N. Özgüven, E. Budak, Analysis and compensation of mass loading effect of accelerometers on tool point FRF measurements for chatter stability predictions, Int. J. Mach. Tool Manu.

doi:10.1016/j.ijmachtools.2010.02.002

[21] T.C. Lim, R. Singh, Vibration transmission throught rolling element bearings. Part I to Part III, J. Sound Vib. 139 (1990) 179-199, 201-225 